\title{
Completely Positive Maps and Entropy Inequalities
}

\author{
Göran Lindblad \\ Department of Theoretical Physics, Royal Institute of Technology, Stockholm \\ Sweden
}

Received May 15, 1974

\begin{abstract}
It is proved that the relative entropy for a quantum system is non-increasing under a trace-preserving completely positive map. The proof is based on the strong subadditivity property of the quantum-mechanical entropy.
\end{abstract}

The object of this note is to prove that the relative entropy functional for a finite quantum system is nonincreasing under a trace-preserving completely positive map of the state space into itself. This theorem is a generalization of an earlier result for expectations [1] (since expectations are completely positive maps [2]) which is in its turn a generalization of a well-known theorem in information theory $[3,4]$. The proof is based on the strong subadditivity property of the quantum-mechanical entropy which was derived recently by Lieb and Ruskai [5] from certain trace inequalities proved by Lieb [6] and, in an alternative way, by Epstein [7].

The physical interest of completely positive maps lies in the theory of measurements and the operational approach to quantum mechanics $[8,9]$. We will give some simple arguments that the operations should be chosen to be completely positive.

Denote by $B(\mathscr{H})$ the bounded operators in a separable Hilbert space $\mathscr{H}$, by $T(\mathscr{H})$ the trace class operators in $\mathscr{H}$ and by $T_{+}(\mathscr{H})$ the positive elements in $T(\mathscr{H})$. Furthermore, let $\mathscr{M}_{n}$ be the algebra of $n \times n$ complex matrices.

Let $A, B \in T_{+}(\mathscr{H})$. Define the operator-valued entropy by $\hat{S}(A)$ $=-A \ln A$.

Let $\lambda \in(0,1)$ and define

$$
\begin{aligned}
& \hat{S}_{\lambda}(A \mid B)=\lambda^{-1}[\hat{S}(\lambda A+(1-\lambda) B)-\lambda \hat{S}(A)-(1-\lambda) \hat{S}(B)] \\
& S_{\lambda}(A \mid B)=\operatorname{Tr} \hat{S}_{\lambda}(A \mid B) .
\end{aligned}
$$

The relative entropy is defined by

$$
S(A \mid B)=\lim _{\lambda \rightarrow 0} S_{\lambda}(A \mid B) .
$$

From Lemma 4 of [10] it follows that this definition is equivalent to that used in $[1,10]$.

We know that $\hat{S}_{\lambda}(A \mid B)$ is positive [10], hence the trace is well-defined, eventually infinite. When $\lambda \downarrow 0, \hat{S}_{\lambda}(A \mid B)$ is monotonously increasing, 
hence the limit $S(A \mid B)$ exists. If $S(A \mid B)<\infty$ then

$$
\hat{S}(A \mid B)=\lim _{\lambda \rightarrow 0} \hat{S}_{\lambda}(A \mid B)
$$

exists as a strong limit. From the properties of the trace follows that

$$
S(A \mid B)=\sup _{P} \sup _{\lambda} \operatorname{Tr} P \hat{S}_{\lambda}(A \mid B)
$$

where $P$ runs over the finite-dimensional projections in $\mathscr{H}$.

A linear positive map $\Phi$ of a $C^{*}$-algebra $\mathscr{A}$ into a $C^{*}$-algebra $\mathscr{B}$ is called completely positive if the induced map

$$
\Phi_{n}=\Phi \otimes I: \mathscr{A} \otimes \mathscr{M}_{n} \rightarrow \mathscr{B} \otimes \mathscr{M}_{n}
$$

is positive for all $n$ [11]. Stinespring [11] has proved that if $\mathscr{A}$ is a $C^{*}$ algebra with unit and if $\mathscr{B}=B(\mathscr{H})$ then there is a *-representation $\pi$ of $\mathscr{A}$ in a Hilbert space $\mathscr{H}^{\prime}$ and a bounded linear map $V: \mathscr{H} \rightarrow \mathscr{H}^{\prime}$ such that $\Phi A=V^{+} \pi(A) V$. Conversely every map of this form is completely positive. It is obvious from the definition that the class of completely positive maps is closed under composition and positive linear combinations. Let $\Phi$ be a positive linear map of $T(\mathscr{H})$ into itself which preserves the trace i.e. $\operatorname{Tr} \Phi A=\operatorname{Tr} A$, all $A \in T(\mathscr{H})$.

Consequently $\Phi$ maps the set of density operators into itself. Assume that $\Phi$ is completely positive with a definition analogous to that above. Then the dual map $\Phi^{+}$defined by

$$
\operatorname{Tr} A \Phi^{+} X=\operatorname{Tr} X \Phi A
$$

for all $X \in B(\mathscr{H}), A \in T(\mathscr{H})$, is a completely positive normal map of $B(\mathscr{H})$ into itself, $\Phi^{+} I=I$ and consequently $\left\|\Phi^{+}\right\|=1$. We can of course consider $\Phi$ to be the dual of $\Phi^{+}$, restricted to the normal linear functionals on $B(\mathscr{H})$.

Denote by $C P(\mathscr{H})$ the set of all completely positive maps $\Phi^{+}$of $B(\mathscr{H})$ into itself and let $C P_{1}(\mathscr{H})$ be the subset satisfying $\left\|\Phi^{+}\right\| \leqq 1$.

The definition of a completely positive map has an interesting physical interpretation. Regard $\Phi$ as an operation $[8,9]$ on the space of normal states, i.e. the density operators, of a finite quantum system (1). Let $\mathscr{M}_{n}$ describe a quantum system (2) which is independent of (1), i.e. the Hilbert space for the combined system (1) $+(2)$ is the tensor product $\mathscr{H}$ $=\mathscr{H}_{1} \otimes \mathscr{H}_{2}$ where $\operatorname{dim} \mathscr{H}_{2}=n$. Then $\Phi_{n}$ describes the resulting operation on the system (1) $+(2)$ provided that $\Phi$ does not influence system (2) directly: $\Phi_{n}\left(A_{1} \otimes A_{2}\right)=\Phi A_{1} \otimes A_{2}$. The condition that $\Phi_{n}$ is positive is obviously necessary in order that states for the total system shall be mapped on states.

It is reasonable to assume that the operations arise through the interaction of the system with an external quantum system i.e. that they are of the form [10]

$$
\Phi A=\operatorname{Tr}_{2} U^{+}(A \otimes B) U
$$


where $A \in T\left(\mathscr{H}_{1}\right), B \in T_{+}\left(\mathscr{H}_{2}\right)$ and $U$ is unitary in $\mathscr{H}_{1} \otimes \mathscr{H}_{2}$. Then $\Phi$ is completely positive. In fact if $\{|i\rangle\},\{\mid k>\}$ are complete orthonormal sets in $\mathscr{H}_{1}$ and $\mathscr{H}_{2}$, respectively, then

satisfies

$$
V_{k}=\sum_{i} I \otimes B^{\frac{1}{2}} U|i k\rangle\langle i|
$$

$$
\Phi A=\Sigma V_{k}^{+}(A \otimes I) V_{k}
$$

which is completely positive by Stinespring's theorem.

We will see below that any trace-preserving completely positive map can be approximated by maps of this type. As examples of completely positive maps occuring in the theory of quantum measurements (apart from the expectations [1]) we can mention the imperfect measurements defined in $[10] \S 5$ and the covariant instruments defined in [12] Theorem 4.

Theorem. If $\Phi$ is a completely positive trace-preserving map of $T(\mathscr{H})$ into itself, then for all $A, B \in T_{+}(\mathscr{H})$

$$
S(\Phi A \mid \Phi B) \leqq S(A \mid B) .
$$

The theorem will be proved via a number of lemmas.

Lemma 1. If $A, A_{n}, B, B_{n} \in T_{+}(\mathscr{H})$ and if $A_{n} \rightarrow A, B_{n} \rightarrow B$ uniformly then

$$
S(A \mid B) \leqq \lim \inf S\left(A_{n} \mid B_{n}\right) .
$$

Proof. Obviously $\hat{S}\left(A_{n}\right) \rightarrow \hat{S}(A)$ and $\hat{S}\left(B_{n}\right) \rightarrow \hat{S}(B)$ uniformly. Consequently $\operatorname{Tr} P \hat{S}_{\lambda}\left(A_{n} \mid B_{n}\right) \rightarrow \operatorname{Tr} P \hat{S}_{\lambda}(A \mid B)$ for every $\lambda$ and every finite dimensional projection $P$. The statement follows from

$$
S(A \mid B)=\sup _{P} \sup _{\lambda} \operatorname{Tr} P \hat{S}_{\lambda}(A \mid B) .
$$

Lemma 2. Let $\mathscr{H}=\mathscr{H}_{1} \otimes \mathscr{H}_{2}$ and put $A_{1}=\operatorname{Tr}_{2} A$ for $A \in T_{+}(\mathscr{H})$. Then, if $A, B \in T_{+}(\mathscr{H})$

$$
S\left(A_{1} \mid B_{1}\right) \leqq S(A \mid B) .
$$

Furthermore, if $A_{1}, B_{1} \in T_{+}\left(\mathscr{H}_{1}\right), A_{2} \in T_{+}\left(\mathscr{H}_{2}\right)$, and $\operatorname{Tr} A_{2}=1$, then $S\left(A_{1} \otimes A_{2} \mid B_{1} \otimes A_{2}\right)=S\left(A_{1} \mid B_{1}\right)$.

Proof. The first statement is the strong subadditivity as formulated in [1] Proposition 2. The second statement is trivial.

Lemma 3. $\Phi^{+}$belongs to an extreme ray in $C P(\mathscr{H})$ if and only if it is of the form $\Phi^{+}(X)=V^{+} X V$ where $V \in B(\mathscr{H})$. The finite linear combinations $\Sigma^{n} V_{i}^{+} X V_{i}, \Sigma^{n} V_{i}^{+} V_{i} \leqq I$ are dense in $C P_{1}(\mathscr{H})$ in the sense of weak operator convergence for every $X$.

Proof. The first statement follows from [13] Corollary 1.4.3, [14] 4.7.22 and the fact that $\Phi^{+}$is normal. The second statement follows from [13] Lemma 1.2.4 and the Krein-Milman theorem.

Lemma 4. Let $\Phi$ be a trace-preserving completely positive map of $T(\mathscr{H})$ into itself, and let $\left\{P_{n}\right\}$ be a family of finite-dimensional projections such that 
$P_{m} \leqq P_{n}$ for $m \leqq n$ and such that $P_{n} \rightarrow I$ strongly when $n \rightarrow \infty$. Then there is a family $\left\{\Phi_{n}\right\}$ of completely positive maps such that $\Phi_{n}$ is trace-preserving on $P_{n} \mathscr{H}$ and $\Phi_{n} A \rightarrow \Phi A$ uniformly for all $A \in T_{+}(\mathscr{H})$.

Proof. First note that $A_{n} \equiv P_{n} A P_{n} \rightarrow A$ uniformly when $n \rightarrow \infty$ ([1], Lemma 4). We can write $A-A_{n}=A_{n}^{+}-A_{n}^{-}$where $A_{n}^{+}$and $A_{n}^{-}$are in $T_{+}(\mathscr{H})$ and $A_{n}^{+} A_{n}^{-}=0$. Obviously $\Phi A_{n}^{+,-} \in T_{+}(\mathscr{H})$ and $\operatorname{Tr} \Phi A_{n}^{+,-} \rightarrow 0$ when $n \rightarrow \infty$. Consequently $\left\|\Phi A_{n}^{+,-}\right\| \rightarrow 0$ and $\left\|\Phi\left(A-A_{n}\right)\right\| \rightarrow 0$ when $n \rightarrow \infty$. Define $\Phi_{n}^{\prime}$ through $\Phi_{n}^{\prime} A=P_{n} \Phi\left(A_{n}\right) P_{n}$. Then

$$
\begin{aligned}
\left\|\Phi A-\Phi_{n}^{\prime} A\right\| & \leqq\left\|\Phi A-P_{n} \Phi(A) P_{n}\right\|+\left\|P_{n} \Phi(A) P_{n}-\Phi_{n}^{\prime} A\right\| \\
& \leqq\left\|\Phi A-(\Phi A)_{n}\right\|+\left\|\Phi A-\Phi A_{n}\right\| \rightarrow 0 \text { when } n \rightarrow \infty .
\end{aligned}
$$

Define $\Phi_{n}$ by $\Phi_{n} A=\Phi_{n}^{\prime} A+V_{n} A V_{n}$ where $V_{n}=\left(P_{n}-\Phi_{n}^{\prime+} I\right)^{\frac{1}{2}}$. Then $\left\|\Phi_{n} A-\Phi A\right\| \rightarrow 0$ and $\operatorname{Tr} \Phi_{n} A=\operatorname{Tr} A$ for all $A$ such that $A=A_{n}$.

Lemma 5. Assume that $\mathscr{H}$ is finite-dimensional and put $\Phi A=\Sigma^{n} V_{i} A V_{i}^{+}$ where $V_{i} \in B(\mathscr{H}), \Sigma^{n} V_{i}^{+} V_{i}=I$ (i.e. $\Phi$ is trace-preserving). Then

$$
S(\Phi A \mid \Phi B) \leqq S(A \mid B) .
$$

Proof. Put $\mathscr{H}^{\prime}=\mathscr{H} \otimes \mathscr{H}_{n}$ where $\operatorname{dim} \mathscr{H}_{n}=n$. Let $\{|i\rangle\}$ be a complete orthonormal set and $|\alpha\rangle$ an arbitrary unit vector in $\mathscr{H}_{n}$. Define

$$
W=\Sigma V_{i} \otimes|i\rangle\langle\alpha| .
$$

Then $W^{+} W=I \otimes P_{\alpha}$ where $P_{\alpha}=|\alpha\rangle\langle\alpha|$ and there is a unitary operator $U$ in $\mathscr{H}^{\prime}$ such that $W=U I \otimes P_{\alpha}$. Consequently

$$
\begin{aligned}
U\left(A \otimes P_{\alpha}\right) U^{+} & =\Sigma V_{i} A V_{j}^{+} \otimes|i\rangle\langle j| \\
\operatorname{Tr}_{2} U\left(A \otimes P_{\alpha}\right) U^{+} & =\Sigma V_{i} A V_{i}^{+}=\Phi A .
\end{aligned}
$$

From Lemma 2 follows that

$S(\Phi A \mid \Phi B) \leqq S\left(U\left(A \otimes P_{\alpha}\right) U^{+} \mid U\left(B \otimes P_{\alpha}\right) U^{+}\right)=S\left(A \otimes P_{\alpha} \mid B \otimes P_{\alpha}\right)=S(A \mid B)$.

Lemma 6. Assume that $\mathscr{H}$ is finite-dimensional and let $\Phi$ be a tracepreserving completely positive map of $B(\mathscr{H})$ into itself. Then

$$
S(\Phi A \mid \Phi B) \leqq S(A \mid B) .
$$

Proof. Lemma 3 implies by duality that $\Phi$ can be approximated by finite linear combinations

$$
\Phi_{n} A=\sum^{n} V_{i} A V_{i}^{+}
$$

with $\Phi_{n}^{+} I=\Sigma V_{i}^{+} V_{i} \leqq I$. Then

$$
\Phi_{n}^{\prime} A=\Phi_{n} A+V_{n+1} A V_{n+1},
$$

where $V_{n+1}=\left(I-\Phi_{n}^{+} I\right)^{\frac{1}{2}}$, is trace-preserving. From Lemma 5 follows that for all positive $A, B$

$$
S\left(\Phi_{n}^{\prime} A \mid \Phi_{n}^{\prime} B\right) \leqq S(A \mid B) .
$$


Due to the fact that $\operatorname{dim} \mathscr{H}$ is finite we obtain uniform convergence $\Phi_{n}^{\prime} A-\Phi A \rightarrow 0$ for every $A$. Obviously

$$
S(\Phi A \mid \Phi B)=\lim _{n} S\left(\Phi_{n}^{\prime} A \mid \Phi_{n}^{\prime} B\right) \leqq S(A \mid B) .
$$

Proof of the Theorem. Choose a set of projections $\left\{P_{n}\right\}$ and define $\Phi_{n}$ as in Lemma 4. From Lemma 6 above and Lemma 3 of [1] we know that for every $n$

$$
S\left(\Phi_{n} A \mid \Phi_{n} B\right) \leqq S\left(A_{n} \mid B_{n}\right) \leqq S(A \mid B) .
$$

From Lemma 2 follows that

$$
S(\Phi A \mid \Phi B) \leqq \lim \inf S\left(\Phi_{n} A \mid \Phi_{n} B\right) \leqq S(A \mid B)
$$

and the theorem is proved.

Acknowledgement. I wish to thank Professor E. H. Lieb for pointing out a mistake in an earlier version of this note.

\section{References}

1. Lindblad,G.: Expectations and entropy inequalities for finite quantum systems. Preprint, February 1974

2. Nakamura, M., Takesaki, M., Umegaki, H.: Kodai Math. Sem. Rep. 12, 82-90 (1960)

3. Kullback, S.: Information theory and statistics. New York: Wiley 1959

4. Umegaki H.: Kodai Math. Sem. Rep. 14, 59-85 (1962)

5. Lieb, E.H., Ruskai, M. B.: J. Math. Phys. 14, 1938-1941 (1973)

6. Lieb, E.H.: Advan. Math. 11, 267-288 (1973)

7. Epstein, H.: Commun. math. Phys. 31, 317-325 (1973)

8. Haag, R., Kastler, D. : J. Math. Phys. 5, 848-861 (1964)

9. Davies, E. B., Lewis, J. T.: Commun. math. Phys. 17, 318-346 (1970)

10. Lindblad, G.: Commun. math. Phys. 22, 305-322 (1973)

11. Stinespring, W.F.: Proc. Am. Math. Soc. 6, 211-216 (1955)

12. Davies, E. B.: J. Funct. Anal. 6, 318-346 (1970)

13. Arveson, W. B.: Acta Math. 123, 141-224 (1969)

14. Dixmier, J.: Les $C^{*}$-algebres et leur representations. Paris: Gauthier-Villars 1969

Communicated by H. Araki
G. Lindblad

Department of Theoretical Physics

Royal Institute of Technology

S-10044 Stockholm 70, Sweden 
